\title{
PISCICOLA HARANTI n.sp. (HIRUDINEA)
}

\author{
Par D. JARRY
}

Cette Piscicole avait été diagnostiquée brièvement dans une note précédente, mais l'auteur propose de l'élever au rang d'une espèce autonome. Une description plus complète et plus adéquate était nécessaire pour confirmer la validité de l'espèce :

$$
\begin{gathered}
\text { Piscicola geometra (L., 1758) } f \text {. haranti (mihi) } \\
=\text { Piscicola haranti n. sp. }
\end{gathered}
$$

Famille des Piscicolidae, Herter, 1935.

Les Piscicolidæ ont un corps cylindrique, très rarement aplati. Etendu, il est souvent plus de 10 fois plus long que large, divisé en deux régions plus ou moins bien individualisées : l'une antérieure ou trachélosome, comprenant les X ou XI somites antérieurs ; l'autre postérieure, souvent plus large, ou urosome, constituée par les somites suivants (1). Les ventouses sont en forme de disque, nettement séparées du corps, avec une trompe protractile s'ouvrant dans le fond de la ventouse antérieure ou orale. Des yeux existent très fréquemment dans la région céphalique, des taches oculiformes sur la ventouse postérieure. Des vésicules contractiles, faisant office de branchies, sont présentes dans la région latérale. La surface dorsale est lisse ou pourvue de papilles sensorielles. L'intestin moyen a deux longs cæcums dirigés vers l'arrière ; ceux-ci peuvent être plus ou moins anastomosés ou fusionnés sur toute leur éten-

(1) Je n'ai pas l'intention de discuter ici de la question excessivement complexe de la métamérie des Piscicolida et des Hirudinées en général. Je m'en tiens à la définition du clitellum admis par les auteurs anglo-saxons : Castle et Moore (1960), Harding et Moore (1927), Meyer (1940), reprise par Scribon et Antrum, Kukenthal, Pawlowski... Le clitellum, région gonflée et moins pigmentée, occupe les somites X-XI-XII-XIII, à l'exception des trois premiers anneaux et des deux derniers. L'orifice mâle se trouve en XI, l'orifice femelle en XII. L'ancienne école, après R. Blanchard (1392), aủmettait IX-X-XI pour le clitellum et les pores génitaux en $\mathrm{X}$ et XI. Le décalage d'un somite est adopté par J. Hoffmann (1956) pour Cystobranchus respirans : il fait alors passer la limite entre régions clitellaire et respiratoire entre les somites XI et XII et non pas XII-XIII et place la première vésicule pulsatile en XII et non pas en XIII, comme M. C. Meyer. 
due. Des diverticules latéraux peu profonds se voient fréquemment, mais plus rarement à l'intestin-postérieur ; 4-6 paires de testicules. Le sperme se transmet au moyen d'un spermatophore; il y a presque toujours une aire copulatrice et un tissu vecteur spécial. Les œufs sont disposés dans un cocon à paroi épaisse, ayant une structure superficielle et une forme distincte selon l'espèce, mais jamais porté par l'individu comme chez les Glossiphonidæ.

Cette très intéressante famille concerne des ectoparasites permanents ou temporaires, principalement de Poissons, accessoirement de Reptiles ou de Crustacés. Elle compte la totalité des Sangsues marines, soit 20-24 genres, et quelques dulçaquicoles, 4-6 genres. Deux seulement sont représentés dans les eaux douces d'Europe : Piscicola et Cystobranchus.

\section{Genre Piscicola (Blainville, 1818) Meyer, emend. 1940.}

Il a été distingué de Hirudo L. par Blainville et peut être considéré comme le type de la famille. Il répond aux caractères énoncés plus haut avec une limitation : toujours six paires de testicules. La bouche s'ouvre au fond de la ventouse orale, en arrière du milieu. La ventouse postérieure est plus grande et plus large que le corps dans sa plus grande largeur. Les somites complets de la région testiculaire ont 14 anneaux et la région respiratoire possède 11 paires de vésicules contractiles.

Le genre compte actuellement six espèces valides et un certain nombre d'autres discutées. Nous retiendrons, avec la majorité des auteurs, en l'absence de données suffisantes ou d'une description complète pour ces dernières (d'ailleurs rangées seulement provisoirement dans le genre):

1. Piscicola geometra (L., 1758). Mondialement répandue dans les régions néarctique et paléarctique, également trouvée en Amérique du Sud. Existe dans toute l'Europe, sauf l'Islande et les pays de même latitude. Forme sténonbiontique et rhéophile.

2. Piscicola olivacea Harding, 1920.

3. Piscicola caeca Kaburaki, 1921. Toutes deux aux Indes.

4. Piscicola punctata (Verrill, 1871).

5. Piscicola milneri (Verrill, 1874). Ces deux dernières dans la région des Grands Lacs d'Amérique du Nord.

6. Piscola salmositica Meyer, 1946. Etat de Washington. 


\section{Piscicola haranti n.sp.}

La découverte en a été faite par le $\mathrm{P}^{\mathrm{r}} \mathrm{H}$. Harant - à qui nous dédions cette espèce nouvelle en témoignage de notre respectueux dévouement et profonde admiration -, à la Source du Lez, aux lieux mêmes où nous avons capturé cette Piscicole 30 ans plus tard (1). «J'ai recueilli aux Sources du Lez, en janvier 1928, deux Piscicoles qui appartiennent peut-être à une espèce nouvelle. Mais, étant donné les grandes variations de l'espèce type et le sens large auquel on la comprend aujourd'hui, j'ai préféré m'abstenir d'un nouveau nom et décrire les formes que j'ai pu examiner. Il s'agit de deux individus de petite taille, dont le tégument est zébré, des espaces pigmentés de bleu alternant avec des espaces parsemés de noir. Malgré leur petite taille, ces Sangsues sont adultes, comme j'ai pu m'en rendre compte par la présence d'ovocytes à maturité dans l'ovaire. Les yeux, la disposition des anneaux dans les somites, les points oculiformes de la ventouse postérieure sont absolument analogues aux formations semblables de $\mathrm{P}$. geometra. Le tégument et les petites dimensions caractérisent donc les Piscicoles prises au Lez. Il s'agit sans doute d'une forme naine, forme géographique de l'espèce type. J'ai récolté ces deux Sangsues sur des pierres plates sur lesquelles elles se déplaçaient à la façon de chenilles arpenteuses. Dans le voisinage, j'ai capturé de jeunes Cobitis barbitula (1) et Cottus gobio. Il est probable que ces Poissons peuvent être les hôtes temporaires de ces Piscicoles. Ces dernières ne contenaient aucun parasite. »

Etudiant les Hirudinées de la Source du Lez depuis plusieurs mois, je découvre en mars 1959 sept individus fixés sur ou sous les pierres, dans une zone très turbulente, entre le premier affluent (bief de l'ancien moulin) et le second. A cet endroit, il existe une plage pierreuse, s'enfonçant assez brusquement, et, vers $50-60 \mathrm{~cm}$. de profondeur, d'autres pierres plus volumineuses dans une végétation de Sium angustifolium et de Scirpus lacustris. Par la suite, tous les individus ayant servi à cette étude ont été retrouvés au

(1) La Source du Lez, puissante résurgence dans les calcaires berriasiens, est située à $18 \mathrm{~km}$. dans le nord de Montpellier. Ses eaux, permanentes en toutes saisons avec un débit variable de $500-10.000$ l./sec., lui viennent d'une capture du Vidourie à Sauve (à $22 \mathrm{~km}$. à vol d'oiseau) par un vaste réseau souterrain sous le massif du Coutach. La population hirudinéenne y est bien représentée : Glossiphonia complanata et Expobdella octoculata CCC., Hemiclepsis marginata C., Theromyzon tessulatum R. et Hæmenteria costata RRR. On trouve la forme type Piscicola geometra dans la végétation immergée du lac inférieur. La faible importance de sa capture n'est probablement pas en rapport avec son importance réelle dans la population.

(1) Actuellement, Nemacheilus barbatulus. 
même endroit; toutefois, il convient de noter que leur nombre n'est jamais très élevé comparativement à celui des autres Sangsues : 3-10 en une heure de pêche ardue (et rendue difficile par le fort courant en ce lieu).

Diagnose : Caractéristiques générales du genre Piscicola. Chaque somite de la région testiculaire a 14 anneaux. Corps presque cylindrique, légèrement effilé vers la partie antérieure. En période reproductrice, clitellum gonflé et plus clair, dilatation suivie d'une constriction indiquant assez nettement la transition entre les régions clitellaire et respiratoire. Le rapport longueur sur largeur est 10/1. Comme l'avait déjà noté $H$. Harant, ce Ver est remarquable par sa faible taille ; les individus s'échelonnent entre $6-14 \mathrm{~mm}$. (R. $20 \mathrm{~mm}$.) et leur poids de 0,60-1,30 $\mathrm{mg}$.

Les ventouses ont la forme et l'aspect types, mais l'antérieure vaut les $2 / 3$ de la postérieure (moyenne 43/68). Deux paires d'yeux distinctes dans la moitié postérieure de la ventouse orale, les organes récepteurs étant placés plus latéralement que chez $P$. geometra. La paire antérieure forme deux taches oblongues sur un angle de $90^{\circ}$ ouvert en arrière. Il n'est pas rare que l'un de ces yeux soit incomplet et paraisse détacher un ocelle en avant; on peut alors croire qu'il y a cinq yeux. La seconde paire est formée d'yeux plus petits, ovoïdes. La ventouse orale déborde en arrière la région du cou, mais sa face dorsale, convexe, est située dans le prolongement de la face dorsale du corps. La ventouse postérieure forme avec le corps un angle ouvert en avant.

Les pigments qui donnent la coloration de fond du Ver examiné vivant sur une plaque sombre - annelures d'un bleu-vert et blanches alternées - sont de trois ordres, comme chez $P$. geometra et Glossophonia complanata (1). Les cellules chromatophores s'étagent dans trois plans différents et se groupent différemment sur les ventouses, les faces dorsale et ventrale. Nous distinguons :

1) Un pigment brun, superficiel, dans des cellules dont le grand axe est perpendiculaire à celui du Ver, très branchues avec des prolongements ramifiés (fig. 3 , en H.).

(1) Autant que nous le sachions, les pigments de $P$. geometra n'ont pas été étuaiiés comme l'ont été ceux de G. complanata. Au premier abord, l'analogie semble frappante, entre un pigment brun responsable des taches sombres et un pigment clair, globuleux, formant les plages blanches. Ce dernier d'ailleurs se révèle double à l'analyse histologique poussée de G. Bobin $(1950)$ : celluies blanches, grosses et réfringentes donnant les taches brillantes superficielles, semblant correspondre à l'une des catégories d'excrétophores des figures de Graf ; cellules à sphérules colorées, jaunes verdâtres, localisées au niveau des cæcums digestifs, avec des irclusions grandes et de taille inégale apparentées au tissu adipeux. 

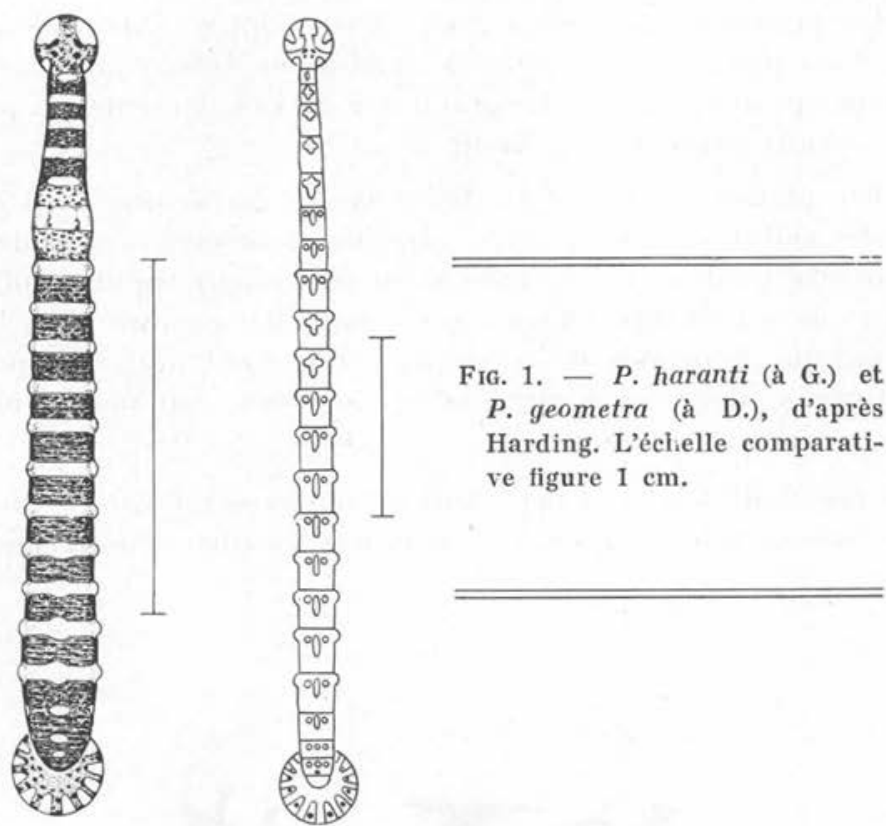

FIG. 1. - P. haranti (à G.) et $P$. geometra (à D.), d'après Harding. L'échielle comparative figure $1 \mathrm{~cm}$.
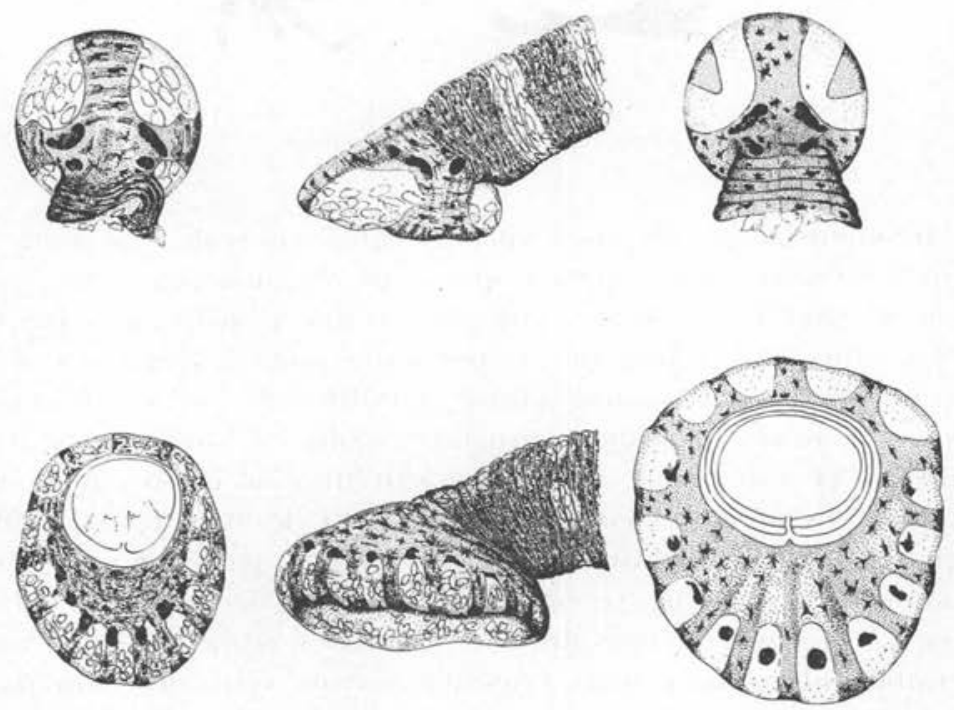

Fig. 2, - Ventouses comparées de P. haranti et $P$. geometra. Les premières ont été dessinées d'après des préparations montées et colorées au carmin acétique ; une correction a été apportée pour les chromatophores après examen sur le vivant: quatre schémas de gauche. Les ventouses de $P$. geometra d'après des exemplaires conservés : à dr., en $\mathrm{H}$. et $\mathrm{B}$. 
2) Un pigment brun-vert ou vert foncé, apparemment plus profond, dans des cellules allongées, parfois en tube contourné, avec de faibles prolongements. Le grand axe de ces éléments est perpendiculaire aux précédents (voir fig. 3, en B.).

3) Un pigment clair, d'un blanc ivoire ou semblable à la cire, dans des cellules volumineuses, presque globuleuses, avec des prolongements faibles, très fragiles et disparaissant rapidement après la mort de l'Annélide. (Raison pour laquelle, comme nous l'avons déjà indiqué à propos de Glossiphonia complanata, l'examen des Hirudinées doit toujours être fait sur le vivant, sur plaque noire et avec un éclairage puissant).

Les pigments bruns et brun-vert se répartissent autour du corps de $P$. haranti pour donner les annelures évoquées, mais sont plus

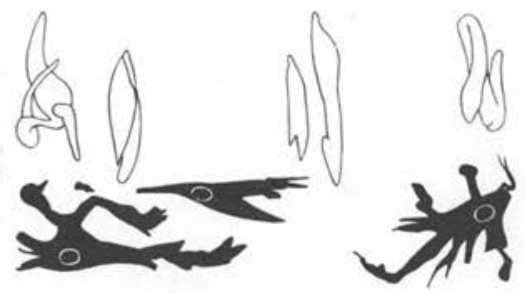

Fig. 3. - Pigments brun (en H.) et brun-vert (en B.) de $P$. haranti.

parcimonieusement disposés sur la région ventrale. Au contraire, le pigment blanc ne se trouve que dans les anneaux clairs, soulignant de plus les vésicules pulsatiles d'une pastille blanche, donnant parfois des taches plus importantes dans la région médiane, pouvant prendre alors une allure moniliforme, au moins sur les derniers somites. De plus, caractère visible à l'œil nu, la bande claire du $\mathrm{XI}^{\circ}$ somite, la sixième à partir du cou, est beaucoup plus large que les autres et coupée latéralement de deux traits sombres (pigment vert-brun). Aussi bien, ce schéma paraît d'une grande constance et on n'observe jamais les rangées de taches si particulières à $P$. geometra : trois dorsales avec les « croix 》 plus ou moins parfaitement réussies, deux latérales et deux ventrales (voir fig. 1).

Quant à la parure des ventouses, elle est également très particulière : tache sombre cruciforme sur la ventouse orale, avec une branche antérieure, deux latérales et une postérieure ; rayons sombres et espaces clairs alternés sur la ventouse postérieure, 6-10 ta- 
ches oculiformes pouvant manquer, jamais dans les plages blanches (disposition rare chez $P$. geometra) (voir fig. 2).

Les vésicules pulsatiles commencent au somite XIII et se continuent métamériquement jusqu'au XXIII ${ }^{\circ}$. L'anus s'ouvre dorsalement entre le troisième et le quatrième anneau, à partir de l'attache de la ventouse postérieure (entre les somites XXVI et XXVII). L'orifice mâle, désigné par ses plis radiaux, a des lèvres formant une légère éminence dans la large bande claire du somite XI. Il semble exister une fossette copulatrice sur le somite XII, mais l'orifice femelle est à peine visible dans un pli transversal, 4-6 anneaux plus en arrière que l'orifice mâle. M. C. Meyer donne les valeurs de cette distance pour les espèces américaines, mais aucun renseignement pour $P$. geometra. Pour nous, elle serait identique ici et là.

Anatomie interne : Le canal alimentaire offre à considérer ses trois parties bien individualisées. L'œsophage est entouré de nombreuses glandes volumineuses sur ses deux tiers antérieurs; celles-ci confluent vers l'extrémité proximale de la trompe qui est courte $(1,10 \mathrm{~mm}$. en moyenne), mais puissamment musculaire. Il débouche dans l'estomac (intestin moyen), dans le XII ${ }^{\circ}$ somite, au niveau d'une cavité cardiale élargie. Cet organe a sept paires d'expansions latérales, entre lesquelles sont logées les six paires de testicules. Ces cæcums sont entiers, n'ont aucune digitation et leurs bords ne sont pas ondulés. A partir du septième diverticule, dans le somite XIX, se dégage l'intestin postérieur, à paroi épaisse, pourvu lui-même de quatre ou cinq diverticules, se continuant par le rectum, le tout placé dorsalement par rapport au cæcum gastrique terminal, membrane fragile qui se continue jusqu'au somite XXV (voir fig. 4).

L'appareil génital révèle l'état de maturité de cette espèce naine. Les testicules, placés de façon intersegmentaire entre les somites XII-XIX, ont un sac renfermant des cellules pigmentaires brunes. De chacun émerge, en avant et latéralement, un vas efferens qui se jette dans le vas deferens latéral. A partir du douzième ganglion, les canaux déférents ont une paroi épaisse, pigmentée, dilatée de place en place. Ils se rapprochent de la ligne médiane, puis se jettent en avant du onzième ganglion, dans les cornes latérales de l'atrium mâle qu'ils abordent par leur angle supéro-interne. Les glandes prostatiques sont abondantes et piriformes ; la partie membraneuse de l'atrium a deux petites cornes paramédianes. L'appareil reproducteur femelle consiste en deux ovaires bursiformes, descendant postérieurement au bord inférieur de la deuxième paire de testicules. Il n'y a pas d'atrium femelle visible. 
Les testicules écrasés livrent les éléments de la spermiogénèse à tous leurs stades de maturité ; la préparation est envahie de morula de spermatogonies et de blastophores massifs avec leur couronne de spermatides ou de spermatozoïdes. Les sacs ovariens libèrent également un grand nombre d'ovocytes.
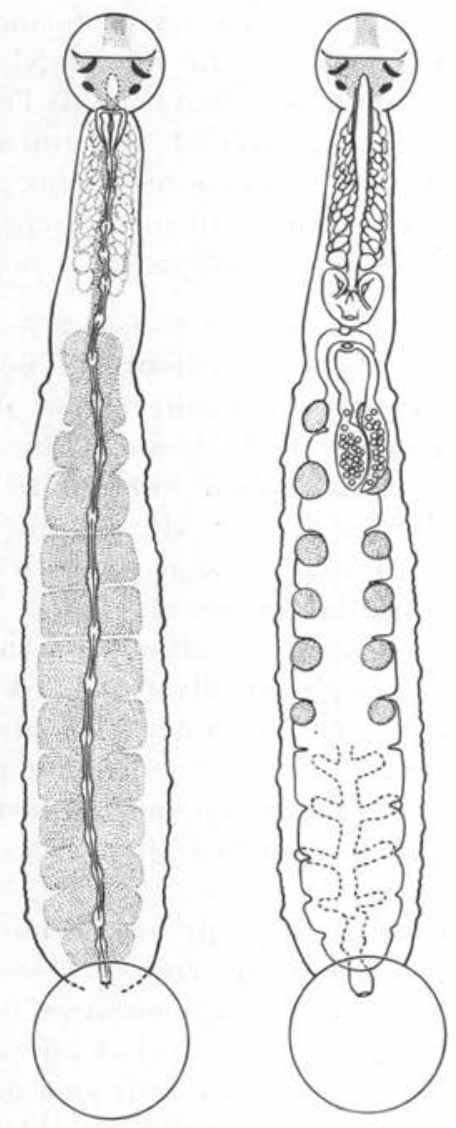

FIG. 4. - Anatomie interne : système nerveux (à G.) ; tube digestif et appareil génital (à D.).

Le système nerveux est classique, avec une masse cérébrale relativement volumineuse, vingt ganglions bien individualisés et un autre postérieur, assez allongé, qui résulte manifestement de la coalescence des plus postérieurs; les distances entre ces derniers sont moins grandes vers les deux extrémités et variables dans la région du clitellum où la chaîne effectue des circonvolutions à large rayon.

Cette anatomie interne, très classique pour le genre, présente de 
grandes ressemblances avec celle de $P$. geometra d'une part (œsophage antérieur, appareil génital), avec l'espèce américaine $P$. salmositica d'autre part (estomac, intestin terminal), sans que l'on veuille naturellement en tirer aucune relation qui serait prématurée...

Ecologie : Cette Piscicole est apparemment une forme rhéocrène ; nous ne la connaissons que de la résurgence du Lez, malgré une prospection poussée, orientée presqu'uniquement sur les Hirudinées et portant sur une centaine de points d'eau dans les environs de Montpellier. Il est assez difficile, en conséquence, de tirer des conclusions sur ce seul biotope, où elle semble fréquenter les pierres beaucoup plus que la végétation où on la rencontre rarement. Les captures s'effectuent toujours au point indiqué plus haut, sur $10 \mathrm{~m}$. de la rivière, en un point où l'eau est rapide, bien qu'encombrée d'une abondante végétation. La Piscicole semble fréquenter aussi bien la face supérieure qu'inférieure des pierres. Très fragile, elle ne survit que quelques heures en aquarium très aéré. Dans un tube traversé par un courant d'eau continu, on arrive à la prolonger quelques jours, pour le temps d'une étude.

Ethologie, comportement : Piscicola haranti est fixée au substrat par sa ventouse postérieure et le corps, resté libre, est déporté par le courant. C'est l'attitude d'attente de $P$. geometra. Toutefois, elle adopte divers comportements en captivité, pour lesquels on peut se demander toujours s'il ne s'agit pas d'une attitude de souffrance. Dans certains cas, l'extrémité céphalique oscille autour de la position moyenne comme l'aiguille d'un dynamomètre ; parfois, c'est la ventouse orale qui est fixée et la Sangsue présente souvent en plus de violents mouvements de torsion, de même qu'elle s'enroule sur elle-même, "se noue », lorsqu'on la retire avec la pierre sur laquelle elle est attachée.

Elle nage parfaitement bien, avec des mouvements ondulatoires dans le plan vertical du déplacement, mais peu longtemps.

Nous n'avons pu assister à sa prise de nourriture, encore qu'expérimentalement on obtienne la fixation sur Phoxinus phoxinus. Les mauvaises conditions de sa survie sont telles que l'on ne peut se prononcer sur son ou ses hôtes normaux, mais, comme H. Harant, nous croyons qu'il ne peut s'agir que d'un Poisson habituel à la mérocénose constituée par les cavités sous les pierres : Cottus gobio CCC, Nemacheilus barbatulus C, mais peut-être Anguilla vulgaris et Lampetra planeri qui peuvent y séjourner occasionnellement.

La reproduction nous est complètement inconnue; aucun des individus que nous possédons n'a une taille inférieure à $6 \mathrm{~mm}$., 
mais les jeunes, possibles dès le mois de mars, doivent passer inaperçus par leur finesse et leur transparence. Quant aux cocons, ils ne peuvent être qu'excessivement petits.

\section{Conclusion}

De ce qui a été dit, il ressort que $P$. haranti occupe une position peu éloignée de $P$. geometra dont elle est peut-être dérivée.

1) Des différences morphologiques externes les distinguent pourtant l'une de l'autre; elles sont rapportées dans le tableau suivant :

TABleaU COMParatif DES CARACTĖRES EXTERnes DE $P$. haranti ET $P$. geometra

\begin{tabular}{c}
\hline \hline \multicolumn{3}{c}{ Piscicola haranti n. sp. } \\
\hline $\begin{array}{l}\text { Longueur de l'adulte : } \\
\text { Poids : } 1-1,80 \mathrm{mg} \text {. }\end{array}$
\end{tabular}

Corps relativement court et cylindrique. Rapport $8-10 / 1$. Clitellum souvent bien reconnaissable (voir fig. 1).

Rapport des ventouses antérieure sur postérieure $=2 / 3$ (voir fig. 2 ).

Yeux gros, bien visibles à l'œil nu, relativement éloignés de la ligne médiane. Aberration 5 fréquente.

Ventouse postérieure avec 12 rayons. Taches oculiformes dans bandelettes foncées.

Parure annelée. A l'œil nu, anneaux bleu-vert et jaune alternés. Clitellum plus clair. La sixième bande claire à partir de l'extrémité céphalique deux fois plus large que les autres, et barrée latéralement. Vésicules pulsatiles soulignées d'une pastille blanche, parfois taches blanches ovalaires médianes sur les derniers somites.

Orifices génitaux séparés de 4-5 segments. Orifice mâle ayant des lèvres formant une légère éminence à la face ventrale de la sixième bande claire. Fossette copulatrice en arrière. Orifice femelle, linéaire, mal visible, dans un pli.
Piscicola geometra L. 1758

Longueur de l'adulte : 40$50 \mathrm{~mm}$. Poids : 6-30 mg.

Forme générale plus élancée. Rapport 12-15/1. Clitellum à peine visible en période de reproduction.

Ce même rapport $=1 / 2$.

Yeux petits, assez rapprochés.

Cette même ventouse, avec $12-14$ rayons. Taches oculiformes dans les plages claires.

Parure caractéristique, faite de « croix » jaunes plus ou moins bien formées sur fond vert. Les taches claires peuvent se résoudre en cinq ordres de lignes longitudinales: trois dorsales, deux latérales et deux ventrales.

Orifices génitaux séparés de cinq segments. Fossette copulatrice toujours reconnaissable par sa couronne de champs tégumentaires soulevés en mamelons. 
2) L'anatomie interne offre moins de termes de comparaison. Toutefois, le grand volume du tube digestif est notoire, ainsi que la configuration très spéciale des diverticules du cæcum gastrique, dont la grande capacité doit assurer une réserve de sang importante pour une aussi petite espèce. L'intestin terminal serait également différent : chez $P$. geometra, il n'existe pas la cinquième paire ébauchée de diverticules, mais il est difficile de trancher dans une dissection aussi fine. L'atrium mâle de $P$. haranti offre à considérer un angle étroit entre ses cornes, disposées comme les branches d'un V, tandis que les déférents s'y continuent insensiblement ; au contraire, celui de $P$. geometra est large, triangulaire, avec une concavité peu profonde entre les cornes qui se terminent en cæcum en avant de l'abouchement des déférents.

3) Les différences de comportement sont aussi évidentes. $P$. geometra, espèce de grande taille, commune, mais difficile à capturer parce que nageant bien, séjourne dans la végétation immergée et se fixe au passage sur des Poissons de pleine eau (Perches, Vairons, Salmonidés, Brochets...) ; P. haranti, rare, localisée (mais demandant à être cherchée ailleurs que le Lez), attend, sous les pierres, le contact des Chabots ou des Loches.

\section{BIBLIOGRAPHIE}

Hafant (H.), 1929. - Les Hirudinées : essai de zoologie médicale et de parasitologie comparée. Imp. Déhan, Montpellier.

Il ARDing (W. A.) et Moone (J. P.), 1927. - Fauna of India. Imp. Taylor and Francis, Londres.

Hoffmann (J.), 1955. - Faune hirudinéenne du Grand-Duché de Luxembourg. Archives, Tome XII, nouv. sér., p. 175-211.

JARRY (D.), 1958. - Les fluctuations dans l'espèce Glossiphonia complanata, Hirudinea. Ann. Soc. hort. hist. nat. Hérault, 98 année, $4^{\circ}$ trim., p. 211217.

JARRY (D.), 1959. - Introduction à 1'étude écologique des hirudinées de la source du Lez. Vie et Milieu, fasc. 3, p. 267-279.

Meyer (M. C.), 1940. - A revision of the leeches (Piscicolida) living on freshwater fishes of North America. Trans. of the Amer. microsc. Society, vol. LIX, $\mathrm{n}^{\circ} 3$, p. 354-376.

Meyer (M. C.), 1946. - Further notes on the leeches (Piscicolidæ) living on fresh-water fishes of North America. Trans. of the Amer. microsc. Society, vol. LXV, n ${ }^{\circ}$, p. 237-249.

Meyer (M. C.), 1946. - A new leech, Piscicola salmositica n. sp. (Piscicolidæ) from steelhead trout, Salmo gairdneri. The Journ. of parasitology, vol. XXXII, $\mathrm{n}^{\circ} 5$, p. 467-476.

Laboratoire d'Histoire naturelle et de Parasitologie médicale de la Faculté de Médecine de Montpellier 